Final (ACCEPTED) VERSion From MARCH 12, 2012

Preprint typeset using $\mathrm{LAT}_{\mathrm{E}} \mathrm{X}$ style emulateapj v. 03/07/07

\title{
COLD GAS ACCRETION BY HIGH-VELOCITY CLOUDS AND THEIR CONNECTION TO QSO ABSORPTION-LINE SYSTEMS
}

\author{
PHILIPP RICHTER \\ Institut für Physik und Astronomie, Universität Potsdam, \\ Haus 28, Karl-Liebknecht-Str. 24/25, 14476 Golm (Potsdam), Germany and \\ Leibniz-Institut für Astrophysik Potsdam (AIP), An der Sternwarte 16, 14482 Potsdam, Germany \\ Final (accepted) version from March 12, 2012
}

\begin{abstract}
We combine H I $21 \mathrm{~cm}$ observations of the Milky Way, M31, and the local galaxy population with QSO absorption-line measurements to geometrically model the three-dimensional distribution of infalling neutral gas clouds ("high-velocity clouds", HVCs) in the extended halos of low-redshift galaxies. We demonstrate that the observed distribution of HVCs around the Milky Way and M31 can be modeled by a radial exponential decline of the mean $\mathrm{H}$ I volume filling factor in their halos. Our model suggests a characteristic radial extent of HVCs of $R_{\text {halo }} \sim 50 \mathrm{kpc}$, a total H I mass in HVCs of $\sim 10^{8} M_{\odot}$, and a neutral-gas-accretion rate of $\sim 0.7 M_{\odot} \mathrm{yr}^{-1}$ for M31/Milky-Way-type galaxies. Using a Holmberglike luminosity scaling of the halo size of galaxies we estimate $R_{\text {halo }} \sim 110 \mathrm{kpc}$ for the most massive galaxies. The total absorption-cross section of HVCs at $z \approx 0$ most likely is dominated by galaxies with total H I masses between $10^{8.5}$ and $10^{10} M_{\odot}$. Our model indicates that the $\mathrm{H}$ I disks of galaxies and their surrounding HVC population can account for 30-100 percent of intervening QSO absorption-line systems with $\log N(\mathrm{HI}) \geq 17.5$ at $z \approx 0$. We estimate that the neutral-gas accretion rate density of galaxies at low redshift from infalling $\mathrm{HVCs}$ is $d M_{\mathrm{HI}} / d t / d V \approx 0.022 M_{\odot} \mathrm{yr}^{-1} \mathrm{Mpc}^{-3}$, which is close to the measured star-formation rate density in the local Universe. HVCs thus may play an important role for the on-going formation and evolution of galaxies.
\end{abstract}

Subject headings: ISM: clouds - quasars: absorption lines - Galaxy: halo

\section{INTRODUCTION}

One crucial aspect of galaxy formation and evolution concerns the continuous infall of intergalactic gas onto galaxies. While it is clear that galaxies do accrete substantial amounts of gas from intergalactic space to power star formation, the exact way of how galaxies get their gas is still a matter of debate. In the conventional sketch of galaxy formation and evolution gas is falling into a dark matter (DM) halo and then is shock-heated to approximately the halo virial temperature (a few $10^{6} \mathrm{~K}$, typically), residing in quasi-hydrostatic equilibrium with the DM potential well (Rees \& Ostriker 1977). The gas then cools slowly through radiation, condenses and settles into the center of the potential where it forms stars as part of a galaxy ('hot mode' of gas accretion). It has been argued, however, that for smaller DM potential wells the infalling gas may radiate its acquired potential energy at much lower temperatures $\left(<10^{5.5} \mathrm{~K}\right.$, typically), so that one speaks of the 'cold mode' of gas accretion (e.g., White \& Rees 1978). For the cold mode of gas accretion the star-formation rate of the central galaxy is directly coupled to its gas-accretion rate (White \& Frenk 1991). Numerical simulations indicate that for individual galaxies the dominating gas-accretion mode depends on the mass and the redshift (e.g., Birnboim \& Dekel 2003; Kereš et al. 2005). The general trend for $z \approx 0$ is that the hot mode of gas accretion dominates for massive galaxies with DM-halo masses $>10^{12} M_{\odot}$, while the cold accretion mode dominates for galaxies with smaller DM-halo masses (e.g., van de Voort et al. 2011).

Independently of the theoretically expected gas-

Electronic address: prichter@astro.physik.uni-potsdam.de accretion mode of galaxies it is known since a long time that galaxies at low and high $z$ are surrounded by large amounts of neutral and ionized gas that partly originates in the IGM. This material is complemented by neutral and ionized gas that is expelled from the galaxies as part of galactic fountains, galactic winds, and from merger processes (see, e.g., Richter 2006 for a review). Because the interplay between these circumgalactic gas components is manifold and the gas physics of such a turbulent multi-phase medium is complex, the circulation of neutral and ionized gas in the inner and outer halos currently cannot be modeled in full detail in hydrodynamical simulations. To improve current models of galaxyevolution models it is of imminent importance to quantify the amount of cool, neutral gas in and around galaxies from observations and search for observational strategies to separate metal-deficient infalling intergalactic gas from metal-enriched gaseous material that is circulating in the circumgalactic environment of galaxies as a result of fountain processes and galaxy mergers.

In the Milky Way and other, very nearby spiral galaxies (e.g., M31), the infall of neutral gas onto to the disks can be observed directly by $\mathrm{H}_{\mathrm{I}} 21 \mathrm{~cm}$ observations of extraplanar gas clouds that move through the halos of these galaxies. For the Milky Way, the so-called "highvelocity clouds" (HVCs) represent the prime candidates for neutral gas that is being accreted onto the Milky Way disk. HVCs represent high-latitude gaseous structures (located in the Galactic halo) observed in $\mathrm{H}$ i $21 \mathrm{~cm}$ emission at high radial velocities, $\left|v_{\text {LSR }}\right|>100 \mathrm{~km} \mathrm{~s}^{-1}$ (e.g.; Wakker \& van Woerden 1998; Richter 2006). Halo clouds with somewhat smaller radial velocities in the range $\left|v_{\mathrm{LSR}}\right|=50-100 \mathrm{~km} \mathrm{~s}^{-1}$ are commonly referred 
to as "intermediate-velocity clouds" (IVCs). Throughout this paper, we will use the expression "HVC" for all neutral halo clouds (including IVCs), if not otherwise stated. The total neutral gas mass of the Milky Way's HVC population is on the order of $10^{8} M_{\odot}$ and the total accretion rate of neutral gas in the form of HVCs has been estimated to be $\sim 0.5 M_{\odot} \mathrm{yr}^{-1}$ (e.g., Wakker et al. 2007, 2008; Wakker 2004). Also M31 exhibits a population of neutral halo clouds in a similar mass range (for simplicity, hereafter also referred to as "HVCs"; Thilker et al. 2004). These observations, together with H I $21 \mathrm{~cm}$ measurements of other nearby galaxies (e.g., Sancisi et al. 2007), imply that HVCs represent a common phenomenon in the local Universe.

Another important method to study the gaseous outskirts of galaxies and their relation to the cosmic web is the analysis of intervening absorption-line systems in optical und ultraviolet (UV) spectra of QSOs (AGN). QSO absorption spectroscopy allows us to detect both neutral and ionized gas in the intergalactic medium and the halos of galaxies over eight orders of magnitude in column density and over more than 90 percent of the age of the Universe. It is therefore a particularly sensitive method to explore the multi-phase nature of circumgalactic gas and its origin (e.g., Bergeron \& Boissé 1992; Steidel 1995; Charlton \& Churchill 1998; Ding et al. 2005; Richter et al. 2011).

In this paper, we combine $\mathrm{H}$ I $21 \mathrm{~cm}$ observations of the HVC population of Milky Way and M31 with $21 \mathrm{~cm}$ data of the local galaxy population and QSO absorption-line measurements to study the three-dimensional distribution of (partly) neutral gas structures in the extended halos of low-redshift galaxies. While several previous studies have linked the Galactic HVC population to intervening QSO absorbers based on various arguments (e.g., Blitz et al. 1999; Charlton, Churchill \& Rigby 2000; Pisano et al.2004; Mshar et al.2007; Schaye, Carswell \& Kim 2007; Narayanan et al. 2008; Richter et al. 2009; Stocke, Keeney \& Danforth 2010; Ribaudo et al. 2011), a detailed geometrical HVC model that connects key observables (column densities, covering fractions, absorber number densities) of HVCs and intervening absorbers with the gas-accretion rates of galaxies at low redshift has not been presented so far. The main goal of the present study is to model the radial distribution of $\mathrm{HVC}$ analogs in halos as a function of galaxy mass and size, determine their absorption-cross section at $z \approx 0$, and estimate the neutral-gas accretion rate of galaxies at low redshift.

We refrain from including $\mathrm{HI} 21 \mathrm{~cm}$ observations of HVCs from other galaxies beyond the Local Group in our study, because these observations are strongly limited in sensitivity for detecting diffuse neutral halo gas (e.g., Oosterloo et al. 2007). In addition, beam-smearing effects are known to smoothen out the true spatial distribution of individual halo clouds in more distant galaxies, so that the observed filling factor of $\mathrm{HI} 21 \mathrm{~cm}$ in these systems does not provide a realistic estimate for the absorption-cross section of this gas.

The paper is organized as follows: in $\S 2$ we discuss $\mathrm{H} \mathrm{I}$ $21 \mathrm{~cm}$ observations of the HVC population of the Milky Way and M31. In $\S 3$ we develop a simple model for the radial distribution of neutral gas in the halos of these galaxies. In $\S 4$ we generalize our HVC model for the local
TABLE 1

Properties $^{\mathrm{a}}$ OF PROMINENT MiLKY WAY HVCs

\begin{tabular}{lcc}
\hline \hline HVC name & $f_{\mathrm{HVC}}^{\mathrm{b}}$ & $\begin{array}{c}D^{\mathrm{c}} \\
{[\mathrm{kpc}]}\end{array}$ \\
\hline Complex A & 0.01 & $8-10$ \\
Complex C & 0.04 & $\sim 10$ \\
Complex H & 0.01 & $>5$ \\
Magellanic Stream & 0.04 & $\sim 50$ \\
Complexes WA, WB & 0.01 & $8-20$
\end{tabular}

a References: Wakker (2001,2004); Wakker et al. (1999, 2007); B.P. Wakker (priv. comm.); Thom et al. (2006, 2008); Gardiner \& Noguchi (1996)

b Sky-covering fraction of HVC gas

c Distance

galaxy population, based on information on the H I mass function of low-redshift galaxies and the absorption-cross section of Damped Lyman $\alpha$ absorbers (DLAs) and Lyman limit systems (LLS) at $z \approx 0$. The relation between HVCs and intervening QSO absorbers is discussed in $§ 5$. In $\S 6$ we present the conclusions from our study.

\section{HIGH-VELOCITY CLOUDS IN THE HALOS OF THE MILKY WAY AND M31}

\subsection{HVCs in the Milky Way}

The HVC population of the Milky Way has a total sky covering fraction of $f_{\mathrm{c}} \approx 0.30$ for column densities $N(\mathrm{H} \mathrm{I}) \geq 7 \times 10^{17} \mathrm{~cm}^{-2}$ and $f_{\mathrm{c}} \approx 0.15$ for column densities $N\left(\bar{H}_{\mathrm{I}}\right) \geq 2 \times 10^{18} \mathrm{~cm}^{-2}$ (Wakker 2004 and references therein). The largest Milky HVC is Complex C, which covers $\sim 1500$ square-degree on the sky $\left(f_{\mathrm{c}} \approx 0.04\right)$. With a low metallicity of $\sim 0.15$ solar (e.g., Fox et al. 2004; Richter et al.2001) and a distance of $D \sim 10$ kpc (Wakker et al. 2007; Thom et al. 2008), Complex C most likely is a cloud that is being accreted from the IGM or from a satellite galaxy. The Magellanic Stream (MS) also covers an area of $\sim 1500$ square-degree, but most likely has a distance as large as $D \sim 50 \mathrm{kpc}$ (Gardiner \& Noguchi 1996). The MS represents a tidal feature expelled from the Magellanic Clouds as they move through the extended Milky Way halo. With its large distance and its stream-like shape the MS is clearly distinct from most of the other Galactic HVCs, which predominantly are less extended and located at distances $<15$ kpc from the disk (Wakker et al. 1999, 2007, 2008; Thom et al. 2006, 2008).

Other prominent Galactic HVCs are Complex A, Complex H, the Anti-Center Cloud, and Complexes WA-WE. Covering fractions and distances for some of these complexes (as far as known) are summarized in Table 1. Because accurate distance information on the Milky Way HVCs is still limited, the total H I mass in HVCs and the H I mass-accretion rate ist not well constrained. The currently available information implies $M_{\mathrm{HI}, \mathrm{HVC}} \sim 3 \times 10^{8} M_{\odot}$ and $d M_{\mathrm{HI}, \mathrm{HVC}} / d t \sim 0.5 M_{\odot} \mathrm{yr}^{-1}$ (Wakker et al. 2007; 2008; Wakker 2004). The H I column densities in HVCs follow a column-density distribution function (CDDF) in the form $f\left(N_{\mathrm{HI}}\right) \propto N_{\mathrm{HI}}^{-\beta}$ with $\beta=1.42$ for $\log N(\mathrm{H} \mathrm{I}) \geq 18$ (Lockman et al. 2002). 

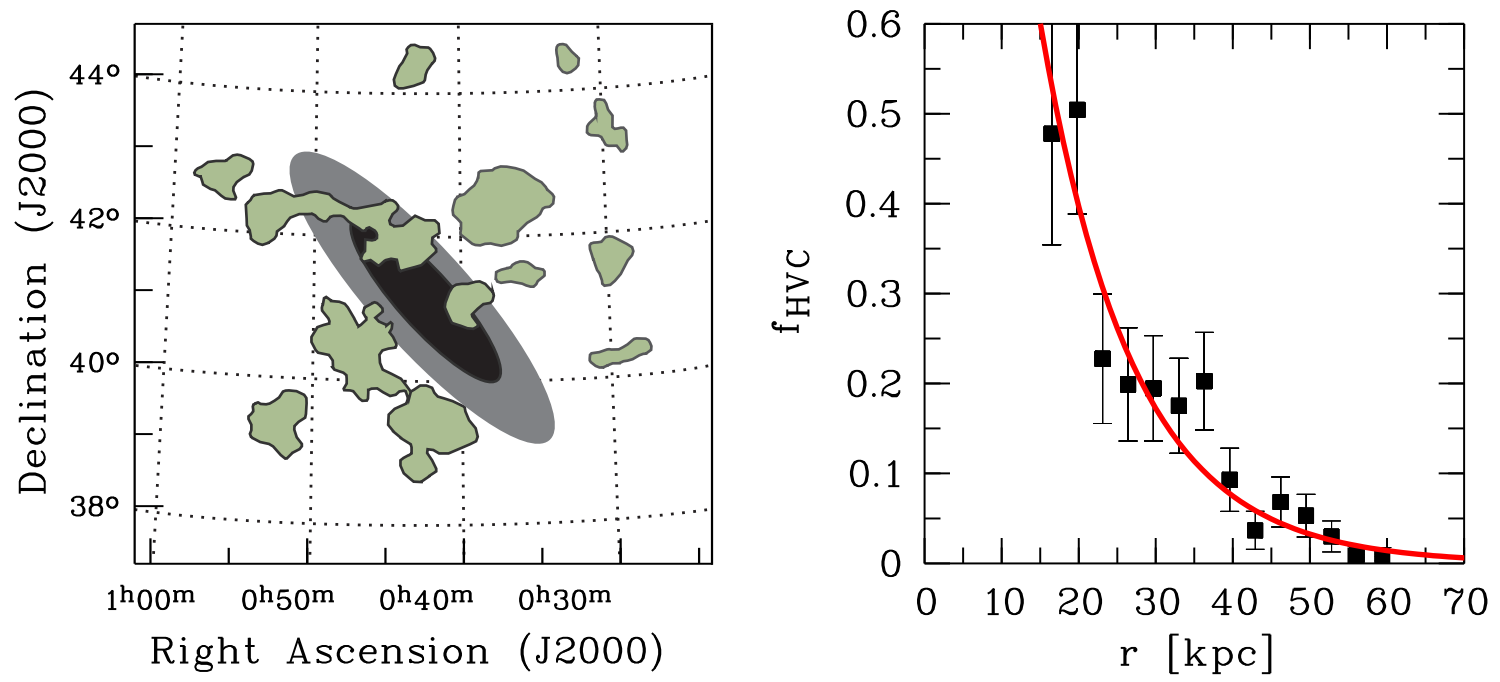

FIG. 1.- Left: distribution of HVCs (green) around M31, adopted from 21cm data of Thilker et al. (2004). The optical disk of M31 is indicated with the black ellipse, the H I disk is shown as gray-shaded ellipse. Note that the shown HVC distribution is incomplete for small radii $(r<15 \mathrm{kpc})$ due to confusion of $\mathrm{H}$ I halo gas with the $\mathrm{H}$ I disk of M31. Right: projected covering fraction of HVC gas around the M31 disk, $f_{\mathrm{HVC}}$, as a function of the projected radius, $r$, based on the HVC distribution shown in the left panel. The covering fraction can be fitted by an exponential in the form $2.1 \exp (-r / 12)$, as indicated with the red solid line.

\subsection{The M31 HVC population}

As has been shown by Braun et al. (2009), M31 has an H I disk that extends to radii of $r \approx 30 \mathrm{kpc}$ for column densities above $\log N(\mathrm{HI}) \geq 20.3$. Beyond $r \approx 30 \mathrm{kpc}$ the H I disk appears to be truncated (Braun et al. 2009; Braun \& Thilker 2004). The HVC population of M31 was studied in detail with the Green Bank Telescope (GBT) by Thilker et al. (2004). These authors detected more than 20 individual HVCs around M31 with H I column densities $\geq 5 \times 10^{17} \mathrm{~cm}^{-2}$ and estimated a total H I mass of the HVCs of $3-4 \times 10^{7} M_{\odot}$ (see also Thilker, Braun \& Westmeier 2005).

In the left panel of Fig. 1 we show the distribution of HVCs around the M31 disk, based on the GBT H I $21 \mathrm{~cm}$ contour map presented by Thilker et al. (2004). The HVC population of M31 reaches out until $\sim 50 \mathrm{kpc}$ with a strongly decreasing $\mathrm{HVC}$ covering fraction towards larger radii. We assume $D=785 \pm 25 \mathrm{kpc}$ as distance for M31 (McConnachie et al. 2005), so that absolute distance estimates derived from angular coordinates are uncertain by $\sim 3$ percent. Some interesting conclusions about the radial distribution of neutral gas around M31 can be drawn from this HVC distribution map. Fig. 1, right panel, shows the radius-dependent projected covering fraction, $f_{\mathrm{HVC}}(r)$, of the HVC population of M31 plotted against $r$ (indicated by the filled boxes), where $r$ is the projected radius. To calculate $f_{\mathrm{HVC}}(r)$ we have resampled the M31 HVC map of Thilker et al. (2004) and have transformed the $(\alpha, \delta)$ coordinate system into a polar coordinate system with coordinates $r$ and $\theta$ centered on M31. For each ring with radius $r$ and thickness $r \pm \Delta r$ the parameter $f_{\mathrm{HVC}}(r)$ then was derived by comparing the area covered by HVC gas with the total ring area. The error bars for $f_{\mathrm{HVC}}(r)$ shown in Fig. 1 have been calculated assuming Poisson-like statistics. Starting from $f_{\mathrm{HVC}} \approx 0.5$ at $r=15 \mathrm{kpc}$ the covering fraction decreases to values less than 0.05 for radii larger than $r=45 \mathrm{kpc}$. This trend for $f_{\mathrm{HVC}}(r)$ can be fitted by an exponential in the form $f_{\mathrm{HVC}}(r)=x \exp (-r / y)$ with $x=2.1 \pm 0.2$ and $y=12.0_{-0.5}^{+0.7}$, as shown by the solid red line in the right panel of Fig. 1. An exponential fit to the extraplanar H I features of M31 was also favored by Braun \& Thilker (2004), who analyzed lower-resolution 21cm data of M31 from the Westerbork Synthesis Radio Telescope (WSRT).

For the inner regions of the M31 halo at $r<15 \mathrm{kpc}$ no HVC data are available. As discussed by Thilker et al. (2004), this does not imply that this region is devoid of HVC material. The lack of data for the disk-halo interface region at $r<15 \mathrm{kpc}$ (Fig. 1, left panel) rather indicates the incompleteness of the HVC map for small radii because of the confusion of neutral halo gas with the HI disk of M31 together with the stringent selection criteria defined by Thilker et al. to unambiguously identify HVC features. In the Milky Way, the disk-halo interface at $r<15 \mathrm{kpc}$ is filled with large amounts of neutral gas that gives rise to $21 \mathrm{~cm}$ emission at intermediate and high velocities (e.g., Wakker 2004). Nearby edgeon galaxies such as NGC 891 also exhibit large amounts of neutral gas in the disk-halo interface region extending several kpc above and below the disk (see Sancisi et al. 2008). $21 \mathrm{~cm}$ measurements of NGC 891 show that the (projected) covering fraction of neutral gas is $f_{\mathrm{HI}}=1$ for vertical distances $d<10 \mathrm{kpc}$ to the midplane of the NGC 891 disk (Oosterloo et al. 2007). If we extrapolate $f_{\mathrm{HVC}}$ for M31 to small radii using the exponential defined above, $f_{\mathrm{HVC}}$ becomes unity for $r \leq 9 \mathrm{kpc}$, in line with the extraplanar gas distribution observed in NGC 891. If we define $r_{3}$ as the radius beyond which the projected covering fraction of HVCs falls below 3 percent, we derive for M31 a value of $r_{3}=50 \pm 6 \mathrm{kpc}$. The $1 \sigma$ error reflects the uncertainties in the exponential parameters for $f_{\mathrm{HVC}}(r)$.

As shown by Braun \& Thilker (2004), the highresolution $21 \mathrm{~cm}$ HVC data of M31 from Thilker et al. (2004) follows a "standard" H I CDDF in the form $f\left(N_{\mathrm{HI}}\right) \propto N_{\mathrm{HI}}^{-\beta}$ with $\beta \approx 1.5$ in the column density range $\log N(\mathrm{HI})=18-20$. The slope is very similar to the one derived for the Milky Way HVCs (Lockman et al. 2002; previous subsection) and is in good agreement with values derived for low-redshift QSO H I absorption-line sys- 
TABLE 2

Model parameters for Milky Way/M31 HVCs

\begin{tabular}{|c|c|c|}
\hline Parameter & Description & Value \\
\hline \multicolumn{3}{|c|}{ Input } \\
\hline $\begin{array}{l}h_{\mathrm{HVC}} \\
f_{\mathrm{v}, 0} \\
\left\langle n_{\mathrm{HI}}\right\rangle \\
z_{\text {IVC }} \\
v_{\text {infall }}\end{array}$ & $\begin{array}{l}\text { scale height of HVC population } \\
\text { central HVC volume fraction } \\
\text { mean H I volume density } \\
\text { upper } z \text {-height limit for IVCs } \\
\text { infall velocity }\end{array}$ & $\begin{array}{l}6.67 \mathrm{kpc} \\
0.0185 \\
0.1 \mathrm{~cm}^{-3} \\
5 \mathrm{kpc} \\
100 \mathrm{~km} \mathrm{~s}^{-1} \text { for HVCs } \\
50 \mathrm{~km} \mathrm{~s}^{-1} \text { for IVCs }\end{array}$ \\
\hline \multicolumn{3}{|c|}{ Output } \\
\hline $\begin{array}{l}f_{\mathrm{HVC}}(r) \\
f_{\mathrm{HVC}}(r) \\
\left\langle f_{\mathrm{IVC}, \mathrm{MW}}\right\rangle \\
\left\langle f_{\mathrm{HVC}, \mathrm{MW}}\right\rangle \\
\left\langle f_{\mathrm{HVC}}\right\rangle \\
r_{3} \\
\langle N(\mathrm{H} \mathrm{I})\rangle \\
M_{\mathrm{HI}, \text { tot }} \\
d M_{\mathrm{HI}, \text { tot }} / d t\end{array}$ & $\begin{array}{l}\text { projected HVC covering fraction for radii } r \geq 9 \mathrm{kpc} \\
\text { projected HVC covering fraction for radii } r<9 \mathrm{kpc} \\
\text { mean IVC covering fraction from inside the sphere } \\
\text { mean HVC covering fraction from inside the sphere } \\
\text { mean HVC/IVC covering fraction from an outside vantage point } \\
\text { radius for which }\left\langle f_{\mathrm{HVC}}\right\rangle \geq 0.03 \\
\text { mean H c column density in HVCs/IVCs from inside the sphere } \\
\text { total neutral gas mass in HVCs/IVCs at } r \leq r_{3} \\
\text { total neutral gas mass accretion rate for gas at } r \leq r_{3}\end{array}$ & $\begin{array}{l}2.1 \exp (-r / 12) \\
1 \\
0.30 \\
0.29 \\
0.21 \\
50 \mathrm{kpc} \\
1.3 \times 10^{19} \mathrm{~cm}^{-2} \\
1.2 \times 10^{8} M_{\odot} \\
0.74 M_{\odot} \mathrm{yr}^{-1}\end{array}$ \\
\hline
\end{tabular}

tems (see Sect. 5.1).

\section{GEOMETRICAL MODELING OF HVCS IN THE LOCAL GROUP}

\subsection{Modeling setup}

To combine the observational information on the HVC population of the Milky Way and M31 we have developed the custom-written numerical code halopath, which is based on a simple geometrical model assuming spherical symmetry. The code allows us to model the radial distribution of gas in the halos of galaxies, its mass distribution and its absorption-cross section from any given vantage point inside and outside the sphere. Because of the unknown size distribution of HVCs we here do no attempt to model individual H I clouds as HVC analogs, but instead consider the volume-filling factor of neutral gas as main input parameter, from which all relevant physical quantities (e.g., projected covering fraction, H I column density, Hi mass) can be easily obtained and compared to observations.

To support the model with the necessary observational data, we assume that the HVC populations of the Milky Way and M31 are identical in a statistical sense (same radial distribution and same volume filling factor of gas with $\log N(\mathrm{HI}) \geq 17.5)$. In view of the similarity of both galaxies in terms of morphology, mass, luminosity, etc. this assumption is justified. As mentioned above, the key parameter that describes the spatial distribution of HVCs in our spherical model is the radius-dependent volume-filling factor of optically thick HVC gas with log $N(\mathrm{HI}) \geq 17.5, f_{\mathrm{v}, \mathrm{HVC}}(R)$, where $R$ is the physical radius (compared to the projected radius $r$ ). Because the projected (area) covering fraction of HVCs in the M31 halo can be described by an exponential (see above), we assume that $f_{\mathrm{v}, \mathrm{HVC}}(R)$ follows an exponential, too, so that we write:

$$
f_{\mathrm{v}, \mathrm{HVC}}=f_{\mathrm{v}, 0} \exp \left(-R / h_{\mathrm{HVC}}\right) .
$$

In this equation, $f_{\mathrm{v}, 0}$ is the volume-filling factor in the center of the sphere and $h_{\mathrm{HVC}}$ is the scale height of the HVC population. Another important parameter in our model is the mean $\mathrm{HI}$ volume density $\left\langle n_{\mathrm{HI}}\right\rangle$ in the $\mathrm{HVC}$ gas, as $\left\langle n_{\mathrm{HI}}\right\rangle$ together with $f_{\mathrm{v}, \mathrm{HVC}}(R)$ determines the neutral gas mass in HVCs per radial bin (= radial volume element), $M_{\mathrm{HI}}(r)=f_{\mathrm{v}, \mathrm{HVC}}(R)\left\langle n_{\mathrm{HI}}\right\rangle \mu m_{\mathrm{H}}$, with $m_{\mathrm{H}}$ as hydrogen mass and $\mu$ as a factor that corrects for the presence of helium and heavy elements in the gas. In addition, $\left\langle n_{\mathrm{HI}}\right\rangle$ determines the mean $\mathrm{H}$ I column density, $\langle N(\mathrm{HI})\rangle$, measured along any given line of sight through the halo, since $\langle N(\mathrm{HI})\rangle=\left\langle n_{\mathrm{HI}}\right\rangle d$, where $d$ is the absorption-path length through the halo. The parameters $\langle N(\mathrm{HI})\rangle$ and $\left\langle n_{\mathrm{HI}}\right\rangle$ are constrained by $21 \mathrm{~cm}$ observations and ionization models of Galactic IVCs and HVCs. Note that in this study we do not model the gas physics in the halo clouds, but consider only the spatial distribution of neutral halo gas, its $\mathrm{H}$ I column density distribution, its total mass, and its infall rate.

To model the sky covering fraction of neutral gas from a vantage point inside the sphere (i.e., to model the projected neutral gas distribution in the Milky Way halo from the position of the sun) we introduce additional constraints based on results from $21 \mathrm{~cm}$ observations of IVCs and HVCs. First, we only consider H I gas as neutral halo gas if it is located at vertical distances $z>600$ $\mathrm{pc}$ from the midplane of the disk (whose orientation can be chosen in the model). Second, we separate neutral halo gas close to the disk (small $z$-heights) from more distant halo clouds via the parameter $z_{\mathrm{IVC}}$. This parameter enables us to distinguish between IVCs $\left(z \leq z_{\mathrm{IVC}}\right)$ and HVCs $\left(z>z_{\mathrm{IVC}}\right)$. Finally, the parameter $v_{\text {infall }}$ defines the infall velocity of the neutral halo gas; $v_{\text {infall }}$ can be a function of $R$ or $z$, or can be chosen to be constant for IVCs and HVCs, respectively. The neutral gas (mass) accretion rate per radial bin then is given by $d M_{\mathrm{HI}}(r) / d t=M_{\mathrm{HI}}(r) v_{\text {infall }} / r$.

\subsection{Modeling results}

Using our geometrical model we are able reproduce the observed properties of the HVC population of the Milky Way and M31 using appropriate values for the above discussed input parameters. Our favorite model is summarized in Table 2. In this model, the scale-height of the HVC population is $h_{\mathrm{HVC}}=6.67_{-0.41}^{+0.53} \mathrm{kpc}$ and the central volume-filling factor of neutral halo gas is $f_{\mathrm{v}, 0}=0.0185 \pm 0.0036$. The errors have been determined 
numerically; they reflect the $1 \sigma$ error range of the exponential parameters for $f_{\mathrm{HVC}}$ in M31 (Sect.2.2). In our model we assume that IVCs and HVCs are separated at a $z$-height of $z_{\mathrm{IVC}}=5 \mathrm{kpc}$, in line with what is known about the distribution of IVC and $\mathrm{HVC}$ distances in the Milky Way halo (Wakker et al. 2007, 2008; Thom et al. 2006, 2008).

Only these three parameters $\left(h_{\mathrm{HVC}}, f_{\mathrm{v}, 0}\right.$, and $z_{\mathrm{IVC}}=$ 5 ) are required to reproduce the observed exponential decline of the projected covering fraction of the HVC population of M31 $\left(f_{\mathrm{HVC}}=2.1 \exp (-r / 12)\right.$ for radii $r \geq 9$ $\mathrm{kpc}$ ) from a vantage point outside the sphere and the observed covering fractions of $\sim 30$ percent for IVCs and HVCs from a vantage point inside the sphere (see Sect.2). Note that for $r<9 \mathrm{kpc}$ the projected covering fraction is set to unity. The characteristic radial extent of the HVC population around both galaxies is $r_{3}=50 \pm 6$ kpc. The mean projected covering fraction of neutral $\mathrm{IVC} / \mathrm{HVC}$ gas in the halo region is $\left\langle f_{\mathrm{HVC}}\right\rangle=0.21 \pm 0.02$.

Observations of $\mathrm{HI}$, metal ions and molecular hydrogen suggest that the neutral hydrogen volume densities in IVCs and HVCs may span a large range over at least three orders of magnitude $\left(10^{-2} \leq n_{\mathrm{HI}} \leq 10 \mathrm{~cm}^{-3}\right.$; e.g., Wakker et al.1999, 2004; Richter et al.2003a, 2003b, 2009; Sembach et al.2001). For our model we adopt a value of $\left\langle n_{\mathrm{HI}}\right\rangle=0.1 \mathrm{~cm}^{-3}$, which is close to the one that has been derived for high-velocity cloud Complex $\mathrm{C}$ (Wakker et al. 1999). We consider this value as a realistic estimate for the volume-averaged mean neutral hydrogen density in IVCs and HVCs. As infall velocity we adopt $v_{\text {infall }}=100 \mathrm{~km} \mathrm{~s}^{-1}$ for HVCs and $50 \mathrm{~km} \mathrm{~s}^{-1}$ for IVCs, assuming that the (vertical) infall velocities towards the disk are comparable to the observed radial velocities of IVCs and HVCs. Since the infall velocity of HVCs is determined by the balance between the gravitational force and the ram-pressure force provided by the surrounding hot coronal gas (e.g., Benjamin \& Danly 1997; Brüns \& Mebold 2004), a more precise modeling of $v_{\text {infall }}$ would also require the modeling of the density distribution of hot halo gas, which is beyond the scope of this study. However, from grid-based hydrodynamical simulations of HVCs Heitsch \& Putman (2009) derive infall velocities that are very similar to the velocities adopted by us.

With the above given values for $n_{\mathrm{HI}}$ and $v_{\text {infall }}$ the mean $\mathrm{HI}$ column density in IVCs and HVCs (from the interior view) is $\langle N(\mathrm{HI})\rangle=1.3 \times 10^{19} \mathrm{~cm}^{-2}$, the total $\mathrm{H}$ I mass in the halo at $r \leq r_{3}$ is $M_{\mathrm{HI} \text {,tot }}=1.2 \times 10^{8} M_{\odot}$, and the total $\mathrm{H}$ I mass accretion rate for gas at $r \leq r_{3}$ is $d M_{\mathrm{HI}, \mathrm{tot}} / d t=0.74 M_{\odot} \mathrm{yr}^{-1}$. These values are in excellent agreement with the observations (see $\S 2$ ).

\section{MODELING OF GALAXY ABSORBERS}

\subsection{Galaxies and their absorption characteristics}

In the previous section we have demonstrated that it is possible to reproduce the statistical properties of the HVC population of the Milky Way and M31 using a model that is based on very simple geometrical assumptions. In the following, we want to generalize our HVC model for the local galaxy population to constrain the absorption cross section of HVCs in the local Universe.

Galaxies and their circumgalactic gaseous environment can be traced by intervening absorption lines of $\mathrm{H}$ I and metal ions in the spectra of distant QSOs and AGN. The strongest intervening neutral-gas absorbers are the so- called Damped Lyman $\alpha$ Absorbers (DLAs), which have $\mathrm{H}$ I column densities $\log N(\mathrm{HI}) \geq 20.3$. These systems contain a substantial fraction of the neutral gas mass in the Universe (Wolfe et al. 1995). Although there still is no consistent picture about the host galaxies of DLAs, observations suggest that a mixed population of galaxies contribute to the absorption-cross section of DLAs at $z \approx 0$ (e.g., Turnshek et al. 2001; Chen \& Lanzetta 2003; Rao et al. 2003). From H i 21cm observations of the local galaxy population Zwaan et al. (2005) concludes, however, that the total DLA cross section at $z \approx 0$ is dominated by the gaseous disks of $L^{\star}$ and sub- $L^{\star}$ galaxies with $\mathrm{H}$ I masses $>10^{9} M_{\odot}$.

Based on Hi $21 \mathrm{~cm}$ observations of the Milky Way, M31, and other nearby galaxies it is expected that neutral gas absorbers in the extended halos of galaxies (i.e., HVC analogs) have $\mathrm{H}$ I column densities below that of DLAs (e.g., Wakker 2004; Thilker et al.2004; Lockman et al.2002; Murphy et al.1995). Using the common absorber classification scheme, halo absorbers therefore are expected to be seen as so-called sub-DLAs $(19.0 \leq \log N(\mathrm{HI})<20.3)$ and Lyman-Limit Systems (LLS; $17.2 \leq \log N(\mathrm{HI})<19.0)$. A large fraction of the LLS at low redshift therefore may represent distant analogs of the HVCs seen around the Milky Way and M31 (Richter et al. 2011).

As for HVCs, the H I column-density distribution function of intervening QSO absorbers at low and high redshift below the DLA column-density limit can be fitted by a power-law in the form $f\left(N_{\mathrm{HI}}\right) \propto N_{\mathrm{HI}}^{-\beta}$. For QSO absorbers, $\beta$ has values between $1-2$, depending on redshift and the column density interval chosen (see Lehner et al.2007). Unfortunately, $\beta$ is poorly constrained for $\log N(\mathrm{H} \mathrm{I})>16$ at $z=0$ due to the limited amount of low-redshift $\mathrm{H}$ I absorption-line data in the UV. In contrast, for high $z$ there exists a large data base that allows us to constrain $\beta$ at a relatively high accuracy (Ribaudo, Lehner \& Howk 2011).

The incidence of intervening DLAs, sub-DLAs and LLS in QSO spectra, usually expressed by the quantity $d \mathcal{N}_{\text {LLS }} / d z$, the number of optically thick H I absorbers per unit redshift, can be obtained from the integration of the $\mathrm{H}$ I CDDF over the appropriate column-density range (in our case $\log N(\mathrm{HI})>17.5$ ). Moreover, $d \mathcal{N}_{\mathrm{LLS}} / d z$ is proportional to the space density of galaxies, $n_{\text {gal }}$, and the mean geometrical cross section of optically thick $\mathrm{H}$, $\left\langle A_{\mathrm{HI}}\right\rangle$, in these galaxies :

$$
\frac{d \mathcal{N}_{\mathrm{LLS}}}{d z}=\int_{N_{\mathrm{HI}} \geq N_{\mathrm{LLS}}} f\left(N_{\mathrm{HI}}\right) d N_{\mathrm{HI}}=\frac{c n_{\mathrm{gal}}\left\langle A_{\mathrm{HI}}\right\rangle}{H(z)} .
$$

We adopt $H(z)=H_{0}\left(\Omega_{\mathrm{m}}(1+z)^{3}+\Omega_{\Lambda}\right)^{1 / 2}, H_{0}=73$ $\mathrm{km} \mathrm{s}^{-1} \mathrm{Mpc}^{-1}, \Omega_{\mathrm{m}}=0.238$, and $\Omega_{\Lambda}=0.762$ (Spergel et al. 2007). We assume that the covering fraction of optically thick $\mathrm{HI}$ gas is $\left\langle f_{\mathrm{HVC}}\right\rangle=1$ in the (inclined) disk of a galaxy and $\left\langle f_{\mathrm{HVC}}\right\rangle<1$ in the surrounding halo, where $\mathrm{HI}$ arises in the form of HVCs. Let $A_{\text {disk }}$ be the geometrical cross section of the (inclined) disk and $A_{\text {halo }}=\pi R_{\text {halo }}^{2}$ the cross section of the (spherical) halo region with radius $R_{\text {halo. }}$. We then can introduce an effective $H V C$ cross section for an individual galaxy, $A_{\mathrm{HVC}, \mathrm{eff}}=\left\langle f_{\mathrm{HVC}}\right\rangle\left(A_{\text {halo }}-A_{\text {disk }}\right)$, so that the total area covered by optically thick $\mathrm{H}_{\mathrm{I}}$ in and around a galaxy is $A_{\mathrm{HI}}=A_{\mathrm{disk}}+A_{\mathrm{HVC}, \mathrm{eff}}$. 

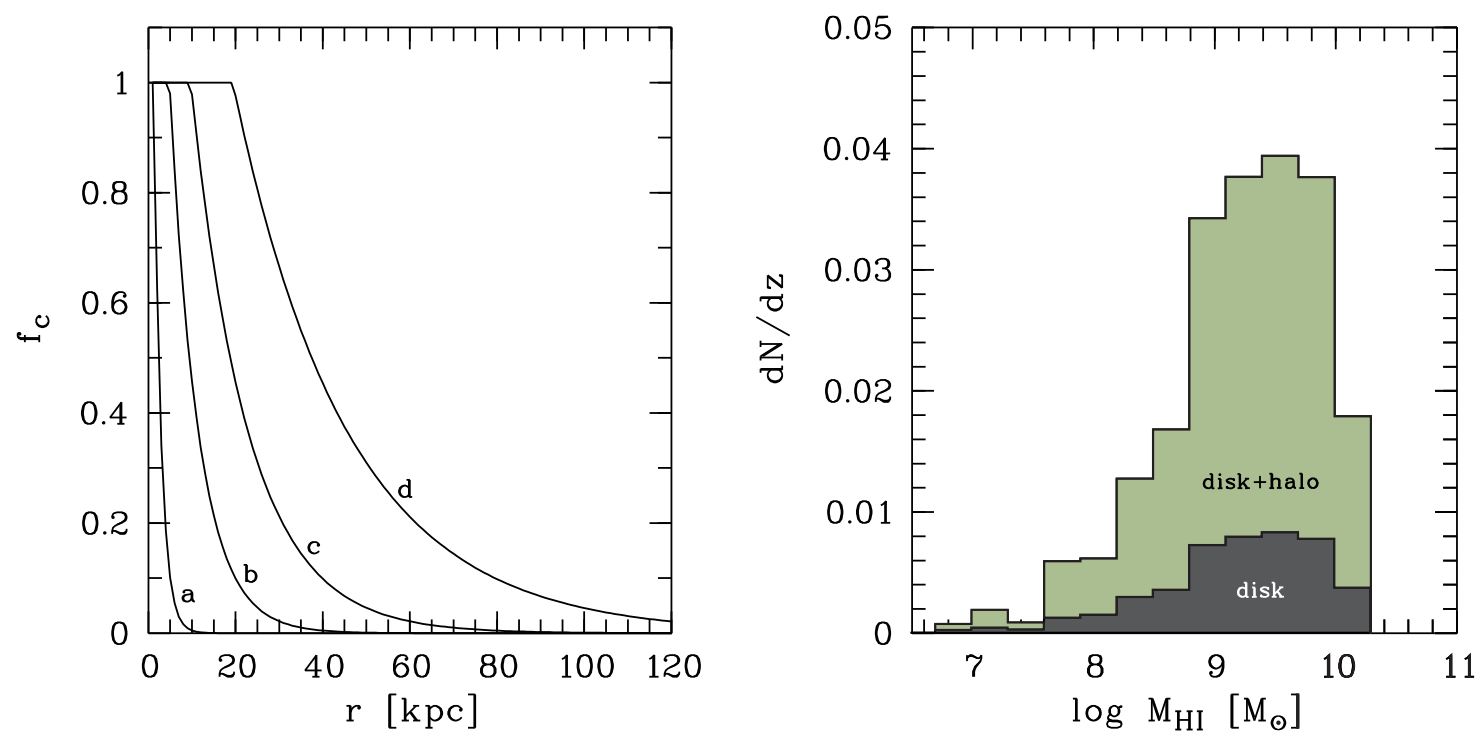

FIG. 2. - Left: projected covering fraction of HVCs around galaxies, $f_{\mathrm{HVC}}$, as a function of the projected radius, $r$, for four different $\mathrm{H}$ I disk masses $\left(\log M_{\mathrm{HI} \text {,disk }}=7.75(\mathrm{a}), 8.95(\mathrm{~b}), 9.55(\mathrm{c}), 10.15(\mathrm{~d})\right.$; see equation 5). Right: expected number density, $d \mathcal{N} / d z$, of optically thick $\mathrm{H}$ I absorbers originating in galaxy disks $(\log N(\mathrm{H} \mathrm{I}) \geq 20.3$; gray-shaded area) and disks plus HVCs $(\log N(\mathrm{H}$ I $) \geq 17.5$; green-shaded area) per galaxy H I mass bin.

From H I $21 \mathrm{~cm}$ measurements of the local galaxy population, Zwaan et al. (2005) have derived a DLA number density per unit redshift of $(d \mathcal{N} / d z)_{\mathrm{DLA}}=0.045 \pm 0.006$, a result that is good agreement with previous estimates (e.g., Rosenberg \& Schneider 2003; $(d \mathcal{N} / d z)_{\text {DLA }}=$ $0.053 \pm 0.013)$. These measurements provide direct information on the individual geometrical cross section (i.e., $\left.A_{\text {disk }}\right)$ of the gaseous disks at $\log N(\mathrm{HI}) \geq 20.3$ in lowredshift galaxies for a large range of galaxy morphologies and luminosities. These H I surveys are, however, not sensitive enough and do not provide sufficient spatial resolution to identify a possibly existing HVC population in the halos of these galaxies.

Based on the above-given relations, a QSO sightline passing through both an $\mathrm{HI}$ disk and HVC gas of a galaxy would show a DLA as absorption signature, while a sightline passing only through an optically thick $\mathrm{HVC}$ would exhibit a sub-DLA or LLS. We then can write for the total number density of $\mathrm{HI}$ absorbers with $\log$ $N(\mathrm{HI}) \geq 17.5$ that trace gas disks of galaxies and their surrounding HVC population:

$$
\left(\frac{d \mathcal{N}}{d z}\right)_{\text {disk }+\mathrm{HVC}}=\left(\frac{d \mathcal{N}}{d z}\right)_{\mathrm{DLA}} \frac{A_{\mathrm{disk}}+A_{\mathrm{HVC}, \mathrm{eff}}}{A_{\text {disk }}} .
$$

Our goal is to estimate $A_{\mathrm{HVC} \text {,eff }}$ from a generalized version of our HVC model for the local galaxy population (for which $A_{\text {disk }}$ is known). This will enable us to estimate $(d \mathcal{N} / d z)_{\text {disk }+ \text { HVC }}$ and link the HVC population at $z=0$ with the $\mathrm{H}$ I column-density distribution function of low-redshift QSO absorbers.

\subsection{On the absorption cross section of neutral gas disks}

Before we start to investigate the absorption crosssection of neutral gas in the halos of galaxies, it is useful to briefly discuss the relation between the absorption cross section, the total H I mass, and the scale-length of neutral gas disks in local galaxies, as derived from $\mathrm{HI}$ $21 \mathrm{~cm}$ surveys. Using $21 \mathrm{~cm}$ data from Arecibo and the Very Large Array (VLA) Rosenberg \& Schneider (2003) have studied in detail these and other properties of 50 nearby galaxies in the context of the low-redshift DLA population. Rosenberg \& Schneider find that the total (inclination-corrected) H I cross section of gas disks in their galaxy sample in the DLA column-density range $(\log N \geq 20.3)$ is given by $A_{\text {disk }}=\pi a b / 4$, where $a$ and $b$ are the major and minor axis parameters (in kpc) derived at the DLA column density limit (see also Rosenberg \& Schneider 2003, their appendix.) They also find a remarkably tight correlation between $A_{\text {disk }}$ and the total H I mass of the disk, $M_{\mathrm{HI}}$ (in solar mass units), as $\log A_{\text {disk }}=\log M_{\mathrm{HI}}-6.82$.

Since the mean value for $b$ in a sample of randomly inclined gas disks is expected to be $0.637 a$, we can write for the mean disk area $\left\langle A_{\text {disk }}\right\rangle \approx 0.16 \pi a^{2}=0.5 a^{2}$ or $\log \left\langle A_{\text {disk }}\right\rangle \approx 2 \log a-0.3$. Combining this with the observed relation between $A_{\text {disk }}$ and $M_{\mathrm{HI}}$, we obtain a relation between the total $\mathrm{H}$ I mass and the disk radius at the DLA limit in the form $\log a=0.5 \log M_{\mathrm{HI}}-3.26$. Finally, Rosenberg \& Schneider (2003) find a (weak) correlation between the H I mass and the J-band luminosity in their sample, $\log M_{\mathrm{HI}}=0.44 \log L_{J}+5.27$, where $L_{J}$ is in solar luminosity units.

\subsection{On the absorption cross section of $H V C s$ surrounding neutral gas disks}

Surveys of the local galaxy population, such as the one presented by Rosenberg \& Schneider (2003), show that galaxies span several orders of magnitude in parameters like H I mass, optical luminosity, and H I disk size. One crucial question that concerns the cross section of neutral gas in the halos of these galaxies is, how the size of the gaseous halo of a galaxy is related to the above listed parameters.

The standard approach to scale the size of a galaxy's gaseous halo with its luminosity is to adopt a Holmberglike luminosity scaling, so that the halo radius is given 
TABLE 3

Properties ${ }^{S}$ of Hi absorbing galaxies

\begin{tabular}{|c|c|c|c|c|c|c|c|}
\hline $\begin{array}{l}\log M_{\mathrm{HI}, \text { disk }} \\
{\left[M_{\mathrm{HI}} \text { in } M_{\odot}\right]}\end{array}$ & $\begin{array}{c}\log A_{\text {disk }} \\
{\left[A \text { in } \mathrm{kpc}^{2}\right]}\end{array}$ & $\begin{array}{c}r_{3} \\
{[\mathrm{kpc}]}\end{array}$ & $\overline{\left\langle f_{\mathrm{HVC}}\right\rangle}$ & $\begin{array}{c}\log A_{\mathrm{HVC}, \mathrm{eff}} \\
{\left[A \text { in } \mathrm{kpc}^{2}\right]}\end{array}$ & $\overline{(d \mathcal{N} / d z)_{\text {disk }+\mathrm{HVC}}}$ & $\begin{array}{l}\log M_{\mathrm{HI}, \mathrm{HVC}} \\
{\left[M_{\mathrm{HI}} \text { in } M_{\odot}\right]}\end{array}$ & $\begin{array}{c}d M_{\mathrm{HI}} / d t \\
{\left[M_{\odot} \mathrm{yr}^{-1}\right]}\end{array}$ \\
\hline $6.7-7.0$ & 0.03 & 2 & 0.35 & 0.41 & $7.1 \times 10^{-4}$ & 3.38 & $5.9 \times 10^{-5}$ \\
\hline $7.0-7.3$ & 0.33 & 3 & 0.23 & 0.88 & $1.9 \times 10^{-3}$ & 4.21 & $3.7 \times 10^{-4}$ \\
\hline $7.3-7.6$ & 0.63 & 4 & 0.21 & 0.97 & $8.4 \times 10^{-4}$ & 4.82 & $1.4 \times 10^{-3}$ \\
\hline $7.6-7.9$ & 0.93 & 7 & 0.15 & 1.51 & $5.9 \times 10^{-3}$ & 5.46 & $5.2 \times 10^{-3}$ \\
\hline $7.9-8.2$ & 1.23 & 9 & 0.17 & 1.73 & $6.1 \times 10^{-3}$ & 5.91 & $1.2 \times 10^{-2}$ \\
\hline $8.2-8.5$ & 1.53 & 13 & 0.18 & 2.05 & $1.3 \times 10^{-2}$ & 6.39 & $3.1 \times 10^{-2}$ \\
\hline $8.5-8.8$ & 1.83 & 19 & 0.18 & 2.40 & $1.7 \times 10^{-2}$ & 6.85 & $7.5 \times 10^{-2}$ \\
\hline $8.8-9.1$ & 2.13 & 27 & 0.19 & 2.70 & $3.4 \times 10^{-2}$ & 7.29 & 0.17 \\
\hline $9.1-9.4$ & 2.43 & 39 & 0.19 & 3.00 & $3.8 \times 10^{-2}$ & 7.73 & 0.40 \\
\hline $9.4-9.7$ & 2.73 & 55 & 0.20 & 3.30 & $3.9 \times 10^{-2}$ & 8.15 & 0.85 \\
\hline $9.7-10.0$ & 3.03 & 78 & 0.20 & 3.61 & $3.8 \times 10^{-2}$ & 8.56 & 1.69 \\
\hline $10.0-10.3$ & 3.33 & 110 & 0.22 & 3.91 & $1.8 \times 10^{-2}$ & 8.95 & 3.09 \\
\hline
\end{tabular}

a Explanations: $r_{3}=$ halo radius beyond which the projected $\mathrm{HVC}$ covering fraction is $\leq 3$ percent; $A_{\mathrm{disk}}=\mathrm{H} \mathrm{I}_{\mathrm{I}}$ disk area; $\left\langle f_{\mathrm{HVC}}\right\rangle=$ mean projected HVC covering fraction for halo region; $A_{\mathrm{HVC} \text {,eff }}=f_{\mathrm{HVC}}\left(A_{\text {halo }}-A_{\text {disk }}\right)$; $(d \mathcal{N} / d z)_{\text {disk }+\mathrm{HVC}}=$ number density of disk/halo absorbers with $\log N(\mathrm{HI}) \geq 17.5$ (per galaxy $\mathrm{H}$ I mass bin); $M_{\mathrm{HI}, \mathrm{HVC}}=$ total neutral gas mass in HVCs per galaxy; $d M_{\mathrm{HI}} / d t=$ neutral gas mass infall rate per galaxy.

by

$$
R_{\text {halo }}(L)=R_{\star}\left(\frac{L}{L^{\star}}\right)^{\delta}
$$

where $\delta \approx 0.2$ and $R_{\star} \approx 110 \mathrm{kpc}$ for B-band luminosities, as derived from analyses of intervening $\mathrm{Mg}$ II absorbers (which trace neutral and ionized gas in disks and halos) and their relation to galaxies (Steidel 1995; Kacprzak et al. 2008). It can be shown that with the above given relations between $L_{J}, M_{\mathrm{HI}}$, and $A_{\text {disk }}$ a Holmberg-like luminosity scaling as given by equation (4) corresponds to a linear scaling of the halo radius with the $\mathrm{H}$ I disk radius. This is because $M_{\mathrm{HI}} \propto L^{0.44}$ (Rosenberg \& Schneider 2003) and $a \propto M_{\mathrm{HI}}^{1 / 2}$, which leads to $R_{\text {halo }} \propto L^{0.22} \propto a$, i.e., a Holmberg-like luminosity scaling with $\delta=0.22$. For the following, we therefore assume a Holmberg-like luminosity scaling with $\delta=0.22$, but we parametrize the halo size over the relation $R_{\text {halo }}(a)=\gamma a$, where $\gamma>1$ and $a$ is the $\mathrm{H}$ I disk radius for $\mathrm{H}$ I column densities above the DLA limit. For M31 $\gamma \approx 1.7$ (see Sect. 2.2).

To characterize the covering fraction of HVCs, $f_{\mathrm{HVC}}$, in galaxy halos, we assume that the exponential decline of $f_{\mathrm{HVC}}$ observed in M31 reflects a general behavior of HVCs in galaxies in the local Universe. We then can express the projected covering fraction of HVCs around galaxies as a function of its $\mathrm{H}$ I disk length in the form

$$
f_{\mathrm{HVC}}(r) \approx\left\{\begin{array}{ll}
1 & : \quad r \leq 0.3 a \\
2.1 \cdot \exp (-2.5 r / a) & : \quad r>0.3 a
\end{array}\right\} .
$$

For M31, $a=30 \mathrm{kpc}$ (Braun et al. 2009; see above). In Fig. 2, left panel, we show $f_{\mathrm{HVC}}(r)$ for four different $\mathrm{H} \mathrm{I}$ masses. In analogy to what has been discussed for the M31 HVC population, we define $r_{3}$ as the halo radius, beyond which $f_{\mathrm{HVC}}$ falls below the 3 -percent level and set $R_{\text {halo }}=r_{3}$. The scaling relation between the disk and halo radius in our model then comes out to $R_{\text {halo }}=1.69 a$ for all halo radii considered (i.e., $\gamma=1.69$ ).

Since $\log a=0.5 \log M_{\mathrm{HI}}-3.26$ (see above), equation (5) allows us to calculate the sizes of neutral gas halos of low-redshift galaxies as a function of their $\mathrm{H}$ I mass and luminosity. It also enables us to predict the cross section and number density of sub-DLAs and LLS that represent HVC analogs, their radial distribution, and estimate the neutral-gas accretion rate of galaxies in the local Universe.

\subsection{Modeling results}

As input for our generalized HVC model we adopt the $\mathrm{H}$ I mass distribution of low-redshift DLAs from Zwaan et al. (2005), as derived from a high-resolution $21 \mathrm{~cm}$ survey of the local galaxy population. Using $21 \mathrm{~cm}$ data from the WRST Zwaan et al. (2005) have studied the H I properties of 355 nearby galaxies and their contribution to the local DLA population. The expected values of $(d \mathcal{N} / d z)_{\text {DLA }}$ for the different $\mathrm{H}$ I masses of the galaxies in their sample are shown as gray-shaded area in the right panel of Fig. 2. Obviously, galaxies with H I masses in the range $\log M_{\mathrm{HI}}=8.8-10.0$ dominate the absorption cross section of DLAs at $z=0$.

Based on these data, we derive for each galaxy H I mass bin the radius and area of the $\mathrm{H} \mathrm{I}$ disk and the $\mathrm{H}$ i halo using the above discussed relations. Note that we here do not take into account the possibility that the neutral gas disks of galaxies significantly extend below the DLA column-density limit. Using our generalized HVC model we then calculate for each mass bin the radius-dependent (projected) HVC covering fraction $\left(f_{\mathrm{HVC}}\right.$; equation 5$)$, the effective HVC cross section $\left(A_{\mathrm{HVC}, \text { eff }}\right)$, and the expected number density of disk + halo $\mathrm{H}$ I absorbers (equation 3). Moreover, our model calculates for each galaxy $\mathrm{H}$ I mass bin the mean projected HVC covering fraction $\left(\left\langle f_{\mathrm{HVC}}\right\rangle\right)$, the total neutral gas mass in $\mathrm{HVCs}\left(M_{\mathrm{HI}, \mathrm{HVC}}\right)$, and the neutral gas mass accretion rate $\left(d M_{\mathrm{HI}} / d t\right)$. For the infall velocities we adopt the values for $v_{\text {infall }}$ for IVCs and HVCs listed in Table 2. The expected number densities $(d \mathcal{N} / d z)_{\text {disk }+\mathrm{HVC}}$ as a function of the galaxy $\mathrm{H}$ I mass are indicated with the green-shaded area in the right panel of Fig. 2. All results are summarized in Table 3 . Based on these results we derive the following relation between the $\mathrm{HI}$ disk mass of galaxies, $\log M_{\mathrm{HI}}$ (in solar units), and the radius of the neutral gas halo, $R_{\text {halo }}$ (in $[\mathrm{kpc}])$ :

$$
\log R_{\text {halo }}=0.5 \log M_{\mathrm{HI}}-3.03 .
$$

If we integrate the values of $(d \mathcal{N} / d z)_{\text {disk }+\mathrm{HVC}}$ listed in 
Table 3 over the entire mass range, we derive a total number density of disk/halo absorbers of $(d \mathcal{N} / d z)_{\text {disk }+\mathrm{HVC}}=$ 0.212 . This value is $\sim 5$ times larger than the number density of DLAs at $z=0$ (Zwaan et al. 2005), suggesting that the absorption-cross section of HVCs with log $N(\mathrm{H} \mathrm{I}) \geq 17.5$ exceeds that of DLAs by a factor of $\sim 4$, on average. As for DLAs, the total absorption cross section of optically thick $\mathrm{H}$ I in disks and halos is dominated by galaxies in the mass range $\log M_{\mathrm{HI}}=8.8-10.0$. As our model indicates, the mean (projected) covering fraction of HVCs in galaxy halos is small, $\left\langle f_{\mathrm{HVC}}\right\rangle=0.2$, typically.

\subsection{Neutral gas accretion-rate density}

Using the mass accretion rates $\left(d M_{\mathrm{HI}} / d t\right)$ listed in Table 3 together with the H I mass function of the local galaxy population (Zwaan et al.2005) we can estimate the neutral gas accretion-rate density of HVCs (mass accretion rate per unit volume) at low redshift. We obtain $d M_{\mathrm{HI}} / d t / d V=0.022 M_{\odot} \mathrm{yr}^{-1} \mathrm{Mpc}^{-3}$. Note that this value is calculated under the assumption that all of the neutral gas in the halos of galaxies is being accreted onto their disks, independently of its origin inside or outside the host galaxies. The above estimate does not include the mass of the ionized gas component of HVCs, which may contribute substantially to the total mass of multi-phase halo clouds (e.g., Fox et al. 2010; Winkel et al. 2011). Also not included are partly neutral gas fragments with masses and angular sizes below the detection limit of $21 \mathrm{~cm}$ HVC surveys. Such structures are known to exist in the Milky Way halo (Richter et al. 2009), but their (total) neutral gas mass most likely is small compared to the large, extended $21 \mathrm{~cm}$ HVCs. The role of ionized gas is further discussed below.

The value of $0.022 M_{\odot} \mathrm{yr}^{-1} \mathrm{Mpc}^{-3}$ is remarkably close to the star-formation rate density at $z=0\left(\dot{\rho}_{\star}=\right.$ $0.01-0.02 M_{\odot} \mathrm{yr}^{-1} \mathrm{Mpc}^{-3}$, as derived from ultraviolet and infrared observational data (Hopkins \& Beacom 2006). Therefore, cold-gas accretion by HVCs possibly plays an important (if not dominating) role in feeding galaxies at $z \approx 0$ with gaseous material to power star formation.

The above given value for the accretion-rate density can also be compared with recent estimates of the "coldmode" gas accretion-rate densities at $z=0$ from cosmological simulations. Using an SPH code, Kere ̌s et al. (2009) find $d M / d t / d V \sim 0.03 M_{\odot} \mathrm{yr}^{-1} \mathrm{Mpc}^{-3}$ for cold gas that never exceeded a maximum temperature of $T_{\max }=2.5 \times 10^{5} \mathrm{~K}$. However, using $\mathrm{SPH}$ simulations with more realistic gas physics van de Voort et al. (2011) derive a much lower "cold-mode" gas accretion-rate density for galaxies at $z=0$ of $d M / d t / d V \sim 0.002 M_{\odot} \mathrm{yr}^{-1} \mathrm{Mpc}^{-3}$, while for the superordinate DM halos the cold-mode accretion-rate is estimated to be $d M / d t / d V \sim 0.01 M_{\odot}$ $\mathrm{yr}^{-1} \mathrm{Mpc}^{-3}$, thus five-times higher. Therefore, only 20 percent of the cold gas that enters the DM halo in their simulation is actually being accreted as cold gas by the central galaxy. Unfortunately, these studies do not provide information on the absorption cross section of all the cold gas in the DM halos and its radial distribution around the galaxies (independently of whether it is being accreted or not), so that a detailed comparison between the simulation results and our HVC model is not possible at this point.

It needs to be mentioned that gas that is considered as "cold" in the cosmological simulations does not necessarily end up as $\mathrm{H}$ I high-velocity gas that is detectable via $21 \mathrm{~cm}$ observations. It is expected that a substantial fraction of the accreted gas that never was heated up to the virial temperature of the host halo remains diffuse and "warm", i.e., at low densities $\left(n_{\mathrm{H}}<10^{-2} \mathrm{~cm}^{-3}\right)$ and intermediate temperatures $\left(T=10^{4}-10^{5} \mathrm{~K}\right)$. The neutral gas fraction in such warm gas is expected to be low, so that it remains unseen in H I $21 \mathrm{~cm}$ emission ("warm" mode of gas accretion; Heitsch \& Putman 2009; BlandHawthorn 2008). The existence of such a warm, ionized gas component in the halos of galaxies is strongly supported by the detection of intermediate- and high-ion absorption (e.g., from Si III, C III, C IV, and Si IV) in the halo of the Milky Way (e.g., Fox et al. 2006; Sembach et al. 1995, 1999) and in the circumgalactic environments of other galaxies (e.g., Ribaudo et al.2011). Also the ionized envelopes of $21 \mathrm{~cm}$ HVC complexes represent significant gas reservoirs that need to be considered for a realistic estimate of the total (neutral and ionized) gas mass that is being accreted by galaxies (e.g., Winkel et al. 2011; Fox et al.2010). The contribution of the ionized gas component to the total gas infall rate is difficult to determine, however, as the infall velocity of ionized gas may be substantially lower than for neutral gas because of hydrodynamical effects that affect the ionized cloud envelopes, such as gas stripping, turbulent mixing, and heat conduction. The same processes also affect the total cloud mass and the neutral gas fraction in HVCs and thus influence the $\mathrm{H}$ I volume filling factor in these clouds. These aspects clearly are best resolved through highresolution hydrodynamical simulations (e.g., Heitsch \& Putman 2009; Vieser \& Hensler 2007).

\section{HVCS AND THEIR RELATION TO INTERVENING QSO ABSORBERS}

\subsection{HVCs and HI Ly $\alpha$ absorbers}

To estimate the contribution of H I HVCs to the number density of optically thick $\mathrm{H}$ I Ly $\alpha$ absorbers at low $z$, it is necessary to know the $\mathrm{HI}$ CDDF for $\log N(\mathrm{HI})>$ 17.5 at low redshift (equation 2). As mentioned above, the CDDF at $z=0$ is poorly constrained for this column density range owing to the fact that high-column density $\mathrm{HI}$ absorbers are rare and that the amount of the QSO absorption-line data in the ultraviolet is limited. Corbelli \& Bandiera (2002) have combined absorption-line data for intermediate redshifts from Bandiera \& Corbelli (2001), low-redshift absorption data from Weymann et al. (1998), and $21 \mathrm{~cm} \mathrm{H} \mathrm{I} \mathrm{emission-line} \mathrm{data} \mathrm{from} \mathrm{Ryan-}$ Weber et al. (2003) to construct the H I CDDF in the range $\log N(\mathrm{HI}) \approx 13-21$. The CDDF presented in Corbelli \& Bandiera (2002) can be fitted by power laws in the ranges $\log N(\mathrm{HI})<18$ and $\log N(\mathrm{HI})>20$, while at $\log N(\mathrm{HI})=18-20$ there seems to be a plateau in $f\left(N_{\mathrm{HI}}\right)$. Since this is exactly the column-density range of relevance for $\mathrm{HVCs}$, these data imply that the total absorption-cross section of HVCs increases only mildly with a decreasing H I cutoff column density. To check the validity of this interesting feature in $f\left(N_{\mathrm{HI}}\right)$ more absorption-line data for the range $\log N(\mathrm{HI})=18-20$ are required. To compare the frequency of sub-DLAs and LLS at low redshift with the HVC absorption cross section obtained from our model we have combined the absorption/emission-line data from Corbelli \& Bandiera 

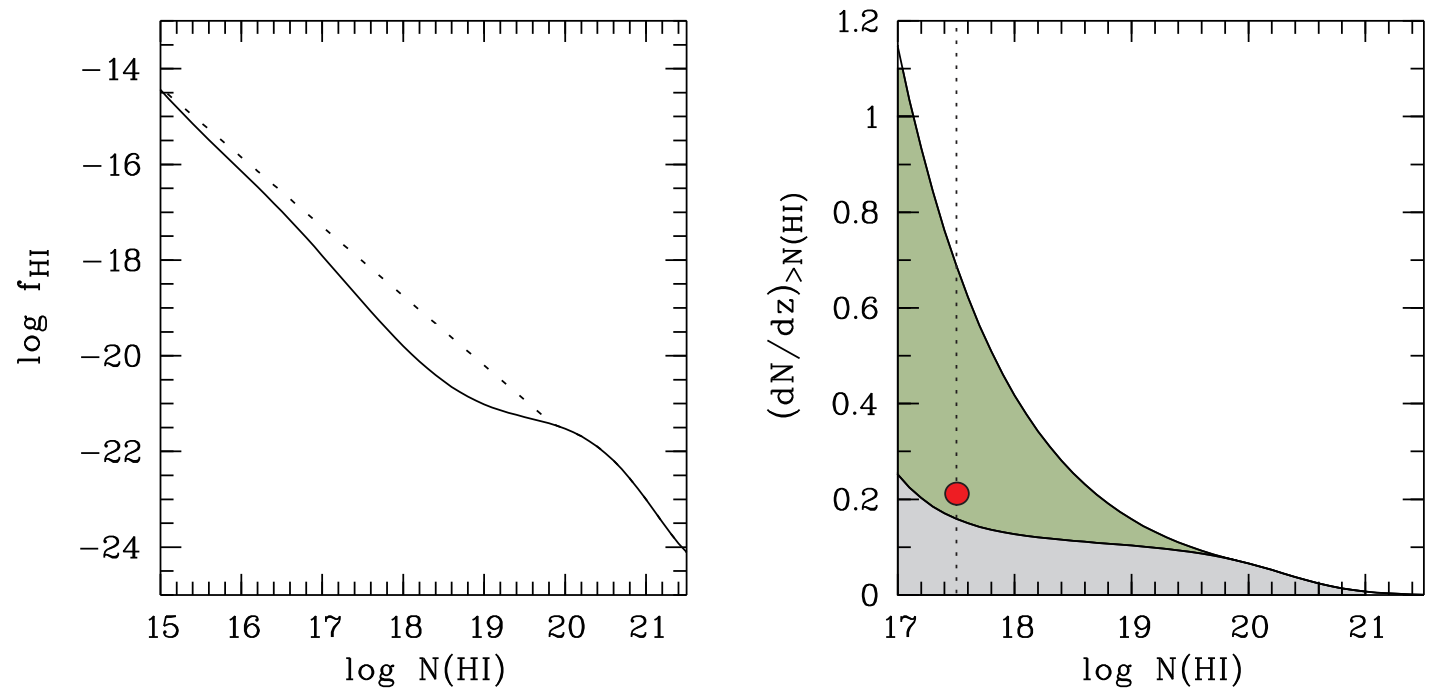

FIG. 3.- Left: H I column-density distribution function (CDDF) at $z \approx 0$, based on results by Corbelli \& Bandiera (2002; solid line), Zwaan et al. (2005; solid line) and Lehner et al. (2007; dashed line). Right: cumulative number density of H I absorbers per unit redshift $\left((d \mathcal{N} / d z)_{>N(\mathrm{HI})}\right)$ as a function of the cutoff $\mathrm{H}$ I column density. The gray-shaded area indicates the allowed $d \mathcal{N} / d z$ range for the CDDF based on the data of Corbelli \& Bandiera (2002) and Zwaan et al. (2005); the gray-shaded area plus the green-shaded area shows the allowed $d \mathcal{N} / d z$ range for the CDDF extrapolated from the Lehner et al. (2007) data. The red filled circle indicates the value for $(d \mathcal{N} / d z)_{\text {disk }+H V C}$ derived from our HVC model.

(2002) and Zwaan et al. (2005) and have constructed a "hybrid" H I CDDF at $z \approx 0$, in Fig. 3, left panel, shown as solid line.

Independent constraints for $f\left(N_{\mathrm{HI}}\right)$ at low redshift come from the study by Lehner et al. (2007), how have analyzed low-redshift Ly $\alpha$ absorbers based on highresolution HST/STIS data. While their study is limited to the column density range $\log N(\mathrm{HI}) \approx 13-16.5$, it is possible to extrapolate their CDDF from the three data points between $\log N(\mathrm{H} \mathrm{I})=14.5-16.5$ (Lehner et al. 2007; their Fig. 14) to $\log N(\mathrm{HI})=20$ with a power law $f(N) \propto N^{-\beta}$, where $\beta \approx 1.3$ (Fig. 3, left panel, dashed line). This extrapolation nicely connects to the $21 \mathrm{~cm}$ data from Zwaan et al. (2005), but lies substantially above the H I CDDF based on the Corbelli et al. results. For the following, we consider both representations of the H I CDDF shown in Fig. 3 as plausible input parameters to estimate the absorption cross section of $\mathrm{H}$ I in sub-DLAs and LLS at $z \approx 0$. In a recent study, Ribaudo, Lehner \& Howk (2011) have compiled a large sample of LLS, most of them located at high redshifts $(z \leq 2.6)$. Interestingly, their data points for $f(N)$ fit well to the Hi CDDF extrapolated from the Lehner et al. (2007) data.

Fig. 3, right panel, shows the (cumulative) number density of $\mathrm{HI}$ absorbers per unit redshift, $(d \mathcal{N} / d z)_{>N(\mathrm{HI})}$, as a function of the cutoff $\mathrm{H}$ I column density, $N(\mathrm{HI})$, dervied from integrating the two representations of the H I CDDF over all column densities larger than $N(\mathrm{HI})$ (equation 2). This plot now can be directly compared to the results from our HVC model. The gray-shaded area indicates the allowed $d \mathcal{N} / d z$ range $(0-100$ percent contribution of HVCs to the H I absorber population) for the hybrid H I CDDF (Corbelli \& Bandiera 2002; Zwaan et al. 2005; left panel, solid line), while the gray-shaded plus green-shaded area shows the allowed $d \mathcal{N} / d z$ range based on the H I CDDF extrapolated from the Lehner et al. (2007) data (left panel, dashed line). The red filled circle indicates the value for $(d \mathcal{N} / d z)_{\text {disk }+ \text { HVC }}$ derived from our HVC model. Thus, our HVC model predicts a value for $d \mathcal{N} / d z$ from $\mathrm{H}$ I disk and halo absorbers that lies slightly above the value expected from the hybrid CDDF, but lies at a $\sim 30$ percent level of $d \mathcal{N} / d z$ predicted by the CDDF extrapolated from the Lehner et al. (2007) data. We conclude that HVCs in our model contribute with $30-100$ percent to the population of $\mathrm{H} \mathrm{I}$ Ly $\alpha$ absorbers with $\log N(\mathrm{HI}) \geq 17.5$ at $z \approx 0$. Most likely, the contribution of HVCs is clearly less than 100 percent, as extended neutral and partly-ionized gas disks with $\log N(\mathrm{H} \mathrm{I})<20.3$ and galaxy outflows (as traced by strong Mg II absorption; see next subsection) contribute to the population of Ly $\alpha$ absorbers in the LLS and subDLA column density range.

A more detailed comparison between the absorption cross section of HVCs and the local $\mathrm{H} \mathrm{I} \mathrm{Ly} \alpha$ absorber population has to await a more precise determination of the $\mathrm{H}_{\text {I }} \mathrm{CDDF}$ at $z \approx 0$ from HST/COS data.

\subsection{HVCs and intervening $M g$ II absorbers}

The Mg II resonant doublet near $2800 \AA$ is commonly used to study neutral and ionized gas in the outskirts of galaxies at $0.3<z<2.2$ (e.g., Bergeron \& Boissé 1991; Charlton \& Churchill 1998; Ding et al.2005). The socalled "strong" Mg II systems represent intervening metal absorbers in QSO spectra that have rest-frame equivalent widths $W>0.3 \AA$ in the $\mathrm{Mg}$ II $\lambda 2796$ line. They are commonly found within $35 h^{-1} \mathrm{kpc}$ of luminous galaxies and thus most likely are related to neutral and ionized gas in the disks and halos of low-redshift galaxies. The number density of strong intervening $\mathrm{Mg}$ II systems in the local Universe is expected to be $(d \mathcal{N} / d z)_{\mathrm{MgII}} \approx 0.5$, as estimated from extrapolating the redshift evolution of strong $\mathrm{Mg}$ II systems in the SDSS data from $z>0.3$ down to $z \approx 0$ (Nestor, Turnshek \& Rao 2005).

The value for $(d \mathcal{N} / d z)_{\mathrm{MgII}}$ is $\sim 2-3$ times higher than $(d \mathcal{N} / d z)_{\text {disk }+ \text { HVC }}$ estimated from our HVC model, 
suggesting a substantially larger absorption cross section of strong Mg II systems compared to HVC H I absorbers with $\log N(\mathrm{HI}) \geq 17.5$. This is expected, because with an ionization potential of $\sim 15 \mathrm{eV}$ Mg II traces both neutral and ionized gas in the disks and halos of galaxies. Kacprzak et al. (2008) estimated a Mg II covering fraction of $\left\langle f_{\mathrm{MgII}}\right\rangle \approx 0.5$ for galaxies and their gaseous halos from a sample of $37 \mathrm{Mg}$ II selected galaxies at intermediate redshift. This is roughly two times the mean value for $\left\langle f_{\text {disk }+ \text { HVC }}\right\rangle$ in our HVC model.

In the Milky Way halo, the Mg II absorption cross section in HVCs has not been determined yet, mostly because of the lack of appropriate high-resolution NUV data. The covering fractions of other low and intermediate ions with strong transitions in the FUV and with ionization potentials comparable to that of $\mathrm{Mg}$ II (e.g., C II, Si II) are larger than the covering fraction of optically thick H I (Richter et al. 2009), but not large enough to explain the high value for $(d \mathcal{N} / d z)_{\text {MgII }}$ in the local Universe. Recent studies suggest, indeed, that many strong $\mathrm{Mg}$ II absorbers at low redshift arise in bipolar outflows and galactic winds (e.g., Bond et al. 2001; Bouché et al. 2011) and thus are related to halo environments with larger $\mathrm{Mg}$ II covering fractions in galaxies that are more actively star-forming than the Milky Way and M31. From our estimate for $(d \mathcal{N} / d z)_{\text {disk }+ \text { HVC }}$ we conclude that the contribution of infalling gas clouds (HVCs) to the absorption cross section of strong $\mathrm{Mg}$ II absorbers most likely is small, but not negligible ( $<35$ percent).

\subsection{HVCs and intervening Ca II absorbers}

Next to the MgII doublet in the near-UV, the two CaII H\&K lines in the optical near $4000 \AA$ represent valuable tracers for neutral gas in the inner and outer regions of galaxies. For instance, Ca II absorption is frequently observed in Galactic HVCs (e.g., Richter, Westmeier \& Brüns 2005; Ben Bekhti et al. 2008) and is used as diagnostic line to derive distance brackets for IVCs and HVCs from optical data (e.g., Wakker et al.2007, 2008; Thom et al.2006, 2008). Based on archival highresolution optical spectra of more than 300 QSOs, obtained with the VLT/UVES spectrograph Richter et al. (2011) have investigated intervening Ca II absorbers at $z \leq 0.5$ and their relation to disk and halo gas components. They derive a number density per unit redshift of $(d \mathcal{N} / d z)_{\text {CaII }}=0.117 \pm 0.044$ for Ca II systems with $\log N(\mathrm{Ca}$ II $) \geq 11.65$, which is roughly 55 percent of the cross section of $\mathrm{H} \mathrm{I}$ HVCs with $\log N(\mathrm{HI}) \geq 17.5$ estimated from our model.

In the Milky Way halo, the covering fraction of Ca II for $\log N(\mathrm{Ca}$ II $) \geq 11.65$ is $\sim 0.2$ (Ben Bekhti et al. 2008), thus $\sim 67$ percent of that of $21 \mathrm{~cm} \mathrm{HVCs} \mathrm{with} \mathrm{log}$ $N(\mathrm{H} \mathrm{I}) \geq 17.5$. As discussed in Richter et al. (2011), Ca II absorbers with $\log N(\mathrm{Ca}$ II $) \geq 11.65$ predominantly trace neutral gas clouds with $\log N(\mathrm{HI}) \geq 18.5$. This is due to the presence of dust in the gas ( $\mathrm{Ca}$ is strongly depleted into dust grains) and the mostly sub-solar metallicities of the absorbers. From these numbers it follows that intervening Ca II absorbers arise only in specific regions in HVCs, where the $\mathrm{H}$ I column density is large enough to compensate for the $\mathrm{Ca}$ dust-depletion and abundance effects.

\section{CONCLUSIONS}

Several previous studies have discussed a possible link between intervening metal-line and $\mathrm{H}$ I absorbers in QSO spectra and the HVC phenomenon in the Milky Way (e.g., Blitz et al.1999; Charlton, Churchill \& Rigby 2000; Mshar et al. 2007; Narayanan et al.2008; Richter et al. 2009; Stocke, Keeney \& Danforth 2010; Ribaudo et al. 2011). In this study, we move this idea forward by bringing together in a quantitative manner the properties of HVCs in the Milky Way and M31, QSO absorptionline statistics, and neutral gas-accretion rates of galaxies in the local Universe. We demonstrate that it is possible to explain the star-formation rate density in the local Universe through the infall of cold gas in the form of HVCs by using a simple geometrical model. In this model we project the observed statistical properties of the H I HVCs in the Local Group onto the local galaxy population, assuming a Holmberg-like luminosity scaling of the halo size. The main results from our modeling are summarized in Tables 2 and 3.

We emphasize at this point that we have not tuned any of the input parameters to fit the HVC cross section to the $\mathrm{H}$ I absorber population or any other above discussed QSO absorber observables. Our generalized HVC model is based solely on observed properties of the HVC population in the Local Group and the local galaxy population without any further assumptions. Note that the exact radial dependence of projected HVC covering fraction, $f_{\mathrm{HVC}}(r)$ (here assumed to be an exponential, based on the M31 data), is unimportant for the total absorption cross section of HVCs, as only the effective area $A_{\mathrm{HVC} \text {,eff }}$ (i.e., the product of total halo area and the mean $\mathrm{HVC}$ covering fraction) is relevant for $(d \mathcal{N} / d z)_{\mathrm{HVC}}$ in a sample of randomly distributed QSO sightlines. However, because the shape of $f_{\mathrm{HVC}}(r)$ reflects the volume-filling factor and thus the mass distribution of neutral gas around galaxies, $f_{\mathrm{HVC}}(r)$ is closely related to the $\mathrm{HI}$ accretion rate of galaxies $d M_{\mathrm{HI}}(r) / d t=M_{\mathrm{HI}}(r) v_{\text {infall }} / r$. Thus, for a more precise estimate of the neutral-gas accretion rate of galaxies at $z=0$ it will be important to constrain the radial distribution of HVCs around other low-redshift galaxies beyond the Local Group using sensitive, highresolution $21 \mathrm{~cm}$ observations. Such observations need to be accompanied by a more precise estimate of the dynamics of neutral gas structures along their infall path through the hot coronal gas in the halos of galaxies of different masses, e.g., from hydrodynamical simulations (Kaufmann et al. 2009; Kereš et al. 2010).

Since most of the gaseous material that is being accreted by galaxies at $z=0$ may be diffuse, ionized gas ("warm accretion"; Bland-Hawthorn 2008) rather than cold, neutral gas in the form of $21 \mathrm{~cm}$ HVCs, it will be important for future studies to investigate the distribution and mass of ionized gas in galaxy halos. Diffuse ionized gas structures in the extended halos of galaxies are expected to have temperatures $T<3 \times 10^{5} \mathrm{~K}$, relatively low gas densities, and low neutral gas fractions (i.e., they remain unseen in $21 \mathrm{~cm}$ HVC surveys). Such structures can be observed best in absorption in the FUV in the lines of low and intermediate ions such as C II, C III, Si II, and Si III (Fox et al.2006; Ribaudo et al.2011). Over the next few years large amounts of such spectral data hopefully will become available from the many ongoing observational campaigns with $\mathrm{HST} / C O S$. Results from these observations can be easily implemented in our halo 
model to predict the ionized-gas accretion rate for lowredshift galaxies.

In conclusion, the increasing amount of information on the distribution and physical properties of gas in the inner and outer halos of galaxies from observations, simulations, and semi-analytic models now can be used to substantially improve our understanding of gas-accretion processes of galaxies in the local Universe. Our study has shown one possible way of how to combine information from the local HVC population and QSO absorption-line systems at $z=0$ to investigate these processes for the local galaxy population. More detailed studies of this kind (e.g., including constraints for ionized halo gas) thus could be of great importance to constrain the role of gas-accretion processes for the ongoing formation and evolution of galaxies at low $z$ and to characterize their connection to the cosmic web.

The author would like to thank Jane Charlton, Glenn Kacprzak, and Bart Wakker for helpful comments and interesting discussions.

\section{REFERENCES}

Bandiera, R. \& Corbelli, E. 2001, ApJ, 552, 386

Ben Bekhti, N., Richter, P., Westmeier, T., \& Murphy, M.T. 2008, A\&A, 487, 583

Benjamin, R.A. \& Danly, L. 1996, ApJ, 481, 764

Bergeron, J. \& Boissé, P. 1991, A\&A, 243, 344

Birnboim, Y. \& Dekel, A. 2003, MNRAS, 345, 349

Bland-Hawthorn, J. 2008, in IAU Symposium 254, The Galaxy Disk in Cosmological Context, astro-ph/08112467 Blitz, L., Spergel, D.N., Teuben, P.J., Hartmann, D., \& Burton, W.B. 1999, ApJ, 514, 818

Bouché, N., Hohensee, W., Vargas, R., Kacprzak, G.G., Martin, C.L., Cooke, J., Churchill, C.W. 2011, astro-ph/11105877 Bond, N.A., Churchill, C.W., Charlton, J.C. \& Vogt, S.S. 2001, ApJ, 557, 761

Braun, R., Thilker, D.A., Walterbros, R.A.M., \& Corbelli, E. 2009, ApJ, 695, 937

Braun, R., \& Thilker, D.A. 2004, A\&A, 417, 421

Brüns, C. \& Mebold, U. 2004, in ASSL, Vol.312, HighVelocity Clouds, ed. van Woerden et al. (Kluwer Academic Publishers), 251

Charlton, J.J., Churchil. C.W., \& Rigby, J.R. 2000, ApJ, 544,702

Charlton, J.C. \& Churchill C.W. 1998, ApJ, 499, 181

Chen, H.-W. \& Lanzetta, K.M. 2003, ApJ, 597, 706

Corbelli, E. \& Bandiera, R. 2002, ApJ, 567, 712

Ding, J., Charlton, J.C., Churchill C.W. 2005, ApJ, 621, 615

Fox, A.J., Wakker, B.P., Smoker, J.V., Richter, P., Savage, B.D., \& Sembach, K.R. 2010, ApJ, 718, 1046

Fox, A.J., Savage, B.D., \& Wakker, B.P. 2006, ApJS, 165, 229

Fox, A.J., Savage, B.D., Wakker, B.P., Richter, P., Sembach, K.R., \& Tripp, T.M. 2004, ApJ, 602, 738

Gardiner, L.T. \& Noguchi, M. 1996, MNRAS, 278, 191

Heitsch, F. \& Putman, M.E. 2009, ApJ, 698, 1485

Hopkins, A.M. \& Beacom, J.F. 2006, ApJ, 651, 142

Kacprzak, G.G., Churchill, C.W., Steidel, C.C., \& Murphy, M.T. 2008, AJ, 135, 922

Kaufmann, T., Bullock, J.S., Maller, A.H., Fang, T., \& Wadsley, J. 2009, MNRAS, 396, 191

Kereš, D., Katz, N., Fardal, M., Davé, R., \& Weinberg, D.H. 2009a, MNRAS, 395, 160

Kereš, D. \& Hernquist, L. 2009b, ApJ, 700, L1

Kereš, D., Katz, N., Weinberg, D.H., \& Davé, R. 2005, MNRAS, 363, 2
Lehner, N., Savage, B.D., Richter, P., Sembach, K.R., Tripp, T.M., \& Wakker, B.P. 2007, ApJ, 658, 680

Lockman, F.J., Murphy, E.M., Petty-Powell, S., \& Urick, V.J. 2002, ApJS, 140, 331

McConnachie, A.W., Irwin, M.J., Ferguson, A.M.N., Ibata, R.A., Lewis, G.F., \& Tanvir, N. 2005, MNRAS, 356, 979

Murphy, E.M., Lockman, F.J., \& Savage, B.D. 1995, ApJ, 447, 642

Mshar, A.C., Charlton, J.C., Lynch, R.S., Churchill, C.W.,\& Kim, T.-S. 2007, ApJ, 669, 135

Narayanan, A., Charlton, J.C., Misawa, T., Green, R.E., \& Kim, T.-S. 2008, ApJ, 689, 782

Nestor, D.B., Turnshek, D.A., \& Rao 2005, ApJ, 628, 637

Oosterloo, T., Fraternali, F., \& Sancisi, R. 2007, ApJ, 134, 1019

Pisano, D.J., Barnes, D.G., Gibson, B.K., Staveley-Smith, L., Freeman, K.C., Kilborn, V.A. 2004, ApJ, 610, L17

Rao, S.M., Nestor, D.B., Turnshek, D.A., Lane, W.M., Monier, E.M., \& Bergeron, J. 2003, ApJ, 595, 94

Rees, M.J., Ostriker, J.P., 1977, MNRAS, 179, 541

Ribaudo, J., Lehner, N., Howk, J.C., Werk, J., Tripp, T.M., Prochaska, J.X., Meiring, J.D. \& Tumlinson J. 2011, ApJ, 743, 207

Ribaudo, J., Lehner, N., \& Howk, J.C. 2011, ApJ, 736, 42

Richter, P., Krause, F., Fechner, C., Charlton, J.C., \& Murphy, M.T. 2011, A\&A, 528, A12

Richter, P., Charlton, J.C., Fangano, A.P.M., Ben Bekhti, N., \& Masiero, J.R. 2009, ApJ, 695, 1631

Richter, P. 2006, Reviews in Modern Astronomy, 19, 31

Richter, P., Westmeier, T., \& Brüns 2005, A\&A, 442, L49

Richter, P., Sembach, K.R. \& Howk, J.C. 2003a, A\&A, 405, 1013

Richter P., Wakker B.P., Savage B.D., \& Sembach K.R 2003b, ApJ, 586, 230

Richter, P., Savage, B.D., Wakker, B.P., Sembach, K.R., Kalberla, P.M.W. 2001, ApJ, 549, 281

Rosenberg, J.L. \& Schneider, S.E. 2003, ApJ, 585, 256

Ryan-Weber, E.V., Webster, R.L., \& Stavely-Smith, L. 2003, MNRAS, 343, 1195

Sancisi, R., Fraternali, F., Oosterloo, T., \& van der Hulst, T. 2008, A\&ARv, 15, 189

Schaye, J., Carswell, R.F., \& Kim, T.-S. 2007, MNRAS, 379, 1169

Sembach, K.R., Howk, J.C., Savage, B.D., Shull, J.M. 2001, AJ, 121, 992

Sembach, K.R., Savage, B.D., Lu, L., \& Murphy, E.M. 1999, ApJ, 515, 108

Sembach, K.R., Savage, B.D., Lu, L., \& Murphy, E.M. 1995, ApJ, 451, 616

Spergel, D.N., Bean, R., Doreé, O., et al. 2007, ApJS, 170, 377

Steidel, C.C., Kollmeier, J.A., Shapley, A.E., Churchill, C.W., Dickinson, M. \& Pettini, M. 2002, ApJ, 570, 526

Stocke, J.T., Keeney, B.A., \& Danforth, C.W. 2010, PASA, 27,256

Thilker, D.A., Braun, R., Westmeier, T. 2005, in: Extraplanar gas, ASP Conference Series, 331, 113

Thilker, D.A., Braun, R., Walterbos, R.A.M., et al. 2004, ApJ, 601, L39

Thom, C., Peek, J.E.G., Putman, M.E., Heiles, C., Peek, K.M.G., \& Wilhelm, R. 2008, ApJ, 684, 364

Thom, C., Putman, M.E., Gibson, B.K., Christlieb, N., Flynn, C., Beers, T.C., Wilhelm, R., Lee, Y.S. 2006, ApJ, 638, L97

Turnshek, D.A., Rao, S., Nestor, D., Lane, W., Monier, E., Bergeron, J., \& Smette, A. 2001, ApJ, 553, 288

van de Voort, F., Schaye, J., Booth, C.M., \& Dalla Vecchia, C. 2011, MNRAS, 414, 2458

Vieser, W. \& Hensler, G. 2007, A\&A, 475, 251 
Wakker, B.P., York, D.G., Wilhelm, R., Barentine, J.C., Richter, P., Beers, T.C., Ivezić, Z., \& Howk, J.C. 2008, ApJ, 672, 298

Wakker, B.P., York, D.G., Howk, J.C., et al. 2007, ApJ, 670, L113

Wakker, B.P. 2004, in ASSL, Vol. 312, High-Velocity Clouds, ed. van Woerden et al. (Kluwer Academic Publishers), 251 Wakker, B.P. 2001, ApJS, 136, 463

Wakker, B.P., Howk, J.C., Savage, B.D. 1999, Nature, 402, 388

Wakker, B.P. \& van Woerden, H. 1998, ARA\&A, 35, 217
Weymann, R.J., Jannuzi, B.T., Lu, L., et al. 1998, ApJ, 506, 1

White, S.D.M. \& Frenk, C.S. 1991, ApJ, 379, 52

White, S.D.M. \& Rees, M.J. 1978, MNRAS, 183, 341

Winkel, B., Ben Bekhti, N., Darmstädter, V., Flöer, L., Kerp, J., \& Richter, P. 2011, A\&A, 533, A105

Wolfe, A.M., Lanzetta, K.M., Foltz, C.B., \& Chaffee, F.H. 1995, ApJ, 454, 698

Zwaan, M.A., van der Hulst, J.M., Briggs, F.H., Verheijen, M.A.W., \& Ryan-Weber, E.V. 2005, MNRAS, 364, 1467 